\title{
Parkinsonismo induzido por trazodona durante o tratamento de hérnia discal lombar: relato de caso
}

Marco de Agassiz Almeida Vasques ${ }^{1}$

Centro Médico Total Care - Setor de Patologias da Coluna Vertebral.

\section{RESUMO}

A utilização de medicações psicoativas vem crescendo ao longo dos anos, sendo essencial o conhecimento de seus efeitos colaterais e interações medicamentosas. O desenvolvimento de distúrbios de movimento associados ao uso dessas substâncias é uma situação bastante desconfortável para o paciente, sendo essencial o diagnóstico adequado mediante forte suspeição. Relata-se o caso de um paciente que desenvolveu sintomas de parkinsonismo durante tratamento de hérnia discal lombar na vigência do uso de trazodona. É dada ênfase aos mecanismos de produção desse fenômeno e à sua condução clínica.

\section{PALAVRAS-CHAVE}

Doença de Parkinson secundária, trazodona, hérnia discal.

\section{ABSTRACT}

Trazodone induced parkinsonism during lumbar disc herniation treatment: case report The use of psychoactive medications has been growing over years, being essential the knowledge of its side effects and interactions. The development of movement disturbances is a very uncomfortable situation for the patient, requiring a high suspicion for adequate diagnosis. A case of a patient who presented symptoms of Parkinsonism during use of Trazodone in the treatment of lumbar disc herniation is reported. Emphasis is given to the biological mechanisms of this phenomenon and its clinical conduction.

\section{KEYWORDS}

Parkinson disease secondary, trazodone, disc herniation. 


\section{Introdução}

A trazodona é um composto de triazolopiridina, muito utilizado por suas propriedades antidepressivas e tranquilizantes, com relativamente poucos efeitos colaterais sobre o sistema nervoso central. ${ }^{1}$ Sua utilização ampla tem levado a uma maior ocorrência de relatos de efeitos colaterais, ${ }^{1-4}$ alguns potencialmente graves. ${ }^{5}$ Apesar disso, tem-se observado um aumento na utilização dessa substância, como em pacientes com fibromialgia. ${ }^{6}$ No presente relato, descreve-se o caso de um paciente que desenvolveu sintomas de parkinsonismo durante acompanhamento de tratamento de hérnia discal lombar na vigência do uso dessa medicação.

\section{Relato do caso}

Paciente de 39 anos, sexo masculino, autônomo, admitido no serviço em julho de 2012 para tratamento de lombociatalgia esquerda iniciada há cinco meses, com limitação funcional para atividades que demandam esforços físicos, VAS 9 e relato de perda de força na perna esquerda. Chegou a receber indicação cirúrgica em outro serviço, porém decidiu não fazer cirurgia inicialmente e iniciar tratamento conservador. Relatou fazer uso de trazodona diariamente há três meses, prescrito por clínico para tratamento de depressão. Fazia uso de anti-inflamatórios e analgésicos, inclusive de tramadol, porém de forma intermitente. O exame físico admissional evidenciava alteração de sensibilidade na face posterior da perna esquerda, reflexo aquiliano esquerdo hipoativo, sinal de Lasègue a 15 graus à esquerda e restrição de mobilidade lombar para flexão. Os estudos de imagem mostravam hérnia discal L5/S1 centro-lateral esquerda. Foi iniciado um programa de reabilitação, com intervenção fisioterápica e controle da dor mediante uso de tramadol. O paciente evoluiu com melhora da sintomatologia dolorosa, com redução da intensidade da dor (VAS 5) e melhora da capacidade funcional, reduzindo a necessidade do uso de analgésicos, que passou a ser esporádico. Em meados de setembro de 2012 apresentou tremores nas mãos, piores à direita, que o impediram de trabalhar. Retornou para atendimento por causa desses novos sintomas, relatando ainda sensação de peso nas pernas e lentidão. Ao exame neurológico, observou-se presença de bradicinesia, discreta hipertonia e tremor do tipo contar notas à direita, em repouso, com restante do exame preservado (os reflexos estavam presentes e simétricos; a sensibilidade e a força estavam normais). Feita a hipótese diagnóstica de parkinsonismo medicamentoso, possivelmente pelo uso há cinco meses, de forma contínua, de trazodona na dose de $150 \mathrm{mg}$ dia. Optou-se pela retirada da droga. Após um período de duas semanas, o paciente retornou para avaliação com relato de melhora dos sintomas. Observou-se ao exame físico desaparecimento dos tremores, da hipertonia e da bradicinesia. Com relação aos sintomas da hérnia discal lombar, manteve a melhora obtida.

\section{Discussão}

Na prática neurocirúrgica, é comum nos depararmos com pacientes que utilizam múltiplas medicações. Particularmente durante o tratamento de patologias degenerativas da coluna, ocorre a prescrição de diversas substâncias psicoativas, como analgésicos, antidepressivos, anticonvulsivantes, entre outras classes medicamentosas. ${ }^{7}$ É importante para o neurocirurgião conhecer os efeitos colaterais dessas medicações, bem como os efeitos da interação entre elas.

A trazodona possui um mecanismo de ação antiserotoninérgico, ${ }^{8}$ podendo inibir circuitos dopaminérgicos de forma secundária, o que explicaria a ocorrência de sintomas extrapiramidais. A literatura tem relatado a ocorrência de distúrbios de movimento associados ao uso de inibidores da recaptação de serotonina. ${ }^{9}$ Albanese et $a .^{2}$ chamam a atenção para a presença de uma sequência de propilpiperazina na estrutura química da trazodona, que se assemelha à encontrada em bloqueadores de canal de cálcio (flunarizina e cinarizina), que já são conhecidos como indutores de parkinsonismo medicamentoso.

$\mathrm{Na}$ condução clínica dos pacientes que desenvolvem esse tipo de sintoma, é fundamental identificar e suspender a medicação causadora do distúrbio ${ }^{10}$ e manter acompanhamento longitudinal para avaliar a efetividade dessas medidas. Muitas vezes o paciente pode apresentar melhora da patologia neurocirúrgica que o levou ao neurocirurgião, porém pode desenvolver complicações relacionadas ao tratamento instituído. A interação com outras especialidades (clínicos, neurologistas, psiquiatras, geriatras) é importante para uma condução mais racional dos pacientes com patologias de tratamento neurocirúrgico.

\section{Referências}

1. Gelenberg AJ. Trazodone (Desyrel): biological therapies in psychiatry. Mass Gen Hosp Newslett. 1983;6:29-30.

2. Albanese A, Rossi P, Altavista MC. Can Trazodone induce parkinsonism? Clin Neuropharmacol. 1988;11(2):180-2. 
3. Fukunishi I, Kitaoka T, Shirai T, Kino K, Kanematsu E, Sato Y. A hemodialysis patient with trazodone-induced parkinsonism. Nephron. 2002;90(2):222-3.

4. Chen HC, Tsai SJ. Trazodone-induced severe headache. Psychiatry Clin Neurosci. 2011;65(7):681-2.

5. Coupland C, Dhiman P, Morriss R, Arthur A, Barton G, Hippisley-Cox J. Antidepressant use and risk of adverse outcomes in older people: population based cohort study. BMJ. 2011;343:d4551.

6. Calandre EP, Morillas-Arques P, Molina-Barea R, RodriguezLopez CM, Rico-Villademoros F. Trazodone plus pregabalin combination in the treatment of fibromyalgia: a twophase, 24-week, open-label uncontrolled study. BMC Musculoskelet Disord. 2011;12:95.

7. Delgado-Lopez PD, Rodriguez-Salazar A, Castilla-Diez JM, Martin-Velasco V, Fernandez-Arconada O. Role of surgery in spinal degenerative disease. Analysis of systematic reviews on surgical and conservative treatments from an evidencebased approach. Neurocirurgia (Astur). 2005;16(2):142-57.

8. Werneck AL, Rosso AL, Vincent MB. The use of an antagonist 5-HT2a/c for depression and motor function in Parkinson disease. Arq Neuropsiquiatr. 2009;67(2B):407-12.

9. Leo RJ. Movement disturbances associated with the use of selective serotonin-reuptake inhibitors. Ann Pharmacother. 1998;32(6):712-4.

10. Lopéz-Sendón JL, Mena MA, de Yébenes JG. Drug-induced parkinsonism in the elderly: incidence, management and prevention. Drugs Aging. 2012;29(2):105-18.

Endereço para correspondência

Marco de A. A. Vasques

SHS, Quadra 04, Bloco G

70314-000 - Brasília, DF, Brasil

Telefone: (61)8138-8538

E-mail: mvasques.ncr@ig.com.br 